\title{
High Single-Mode Power Conversion Efficiency Vertical-Cavity Top-Surface-Emitting Lasers
}

\author{
K. L. Lear and S. A. Chalmers
}

\begin{abstract}
We report advances in the power conversion (wallplug) efficiency of vertical-cavity top-surface-emitting lasers. The devices were fabricated from molecular beam epitaxial layers using deep proton implants to define gain-guided lasers. The epitaxial structure included low resistance, piecewise linearly graded n-type and p-type mirrors, a triple $\mathrm{In}_{0.2} \mathrm{Ga}_{0.8}$ As quantum-well active region, and a delta-doped contact layer. Power conversion efficiencies as high as $\mathbf{1 2 . 7 \%}$ for continuous-wave single-mode operation were measured after several hours of device operation.
\end{abstract}

$\mathrm{V}^{\mathrm{s}}$ ERTICAL-cavity surface-emitting lasers (VCSEL's) provide many advantages over edge-emitting lasers. Their suitability for integration into two-dimensional (2-D) arrays is often noted, but they also compare favorably in many respects even when considered as single devices. They operate in a single longitudinal mode; they emit a low-divergence, circular beam; their fabrication is simpler since facet formation is not required; and devices can be tested at the wafer level. While these attributes make VCSEL's attractive for many applications, their power conversion (wallplug) efficiency is presently much lower than that of edge-emitting lasers, which have reported power conversion efficiencies up to $60 \%$ [1]. Improvements in VCSEL efficiency will relax system power requirements for device applications and reduce excessive heating, thus allowing increased packing density and improved reliability.

Recently, multimode VCSEL's have been reported with room temperature, continuous wave $(\mathrm{CW})$ power conversion efficiencies up to $17.3 \%$ for bottom-emitting devices in which the light output passes through the substrate [2], [3]. This geometry allows for contacts and heatsinking above the entire active region and is well suited for long-wavelength operation. In contrast, the necessity of an aperture in the top metallic contact of top-emitting VCSEL's [4] increases their resistance, thereby reducing the efficiency of devices with this contact geometry [5]. However, top-emitting VCSEL's are desirable for applications where the substrate is absorbing at the operating

Manuscript received May 25, 1993. This work was supported by the U.S. Department of Energy under Contract DE-AC04-76DP00789.

The authors are with the Photonics Research Department, Sandia National Laboratories, Albuquerque, NM 87185-5800.

IEEE Log Number 9211414. wavelength, e.g., 850-nm GaAs/AlGaAs devices or 650-nm visible VCSEL's [6]. Top-emitting VCSEL's are also more easily tested and packaged and are adaptable to certain 2-D coherent array phase correction techniques [7].

In this letter, we report $\mathrm{CW}$ power conversion efficiencies as high as $12.7 \%$ for top-surface-emitting devices in single-mode operation without special heatsinking or packaging. Previously reported CW power conversion efficiencies for top-emitting VCSEL's are below 10\% [8]. Several parameters affect power conversion efficiency in semiconductor lasers including internal quantum efficiency, threshold, distributed and mirror optical losses, and series resistance [9]. The last parameter has been extremely high for VCSEL's until recently due to potential barriers at the heterointerfaces of distributed Bragg reflector (DBR) mirrors. In order to realize more efficient top-emitting VCSEL's, we have primarily focused on decreasing the series resistance of mirror stacks, as well as contacts to implanted material. A method for grading DBR heterojunctions that resulted in specific resistivities as low as $1.8 \times 10^{-5} \Omega \cdot \mathrm{cm}^{2}$ for a 20 period p-type mirror with a Be doping concentration of $5 \times 10^{18} \mathrm{~cm}^{-3}$ grown by molecular beam epitaxy (MBE) was previously reported [10]. The quality of these mirrors has been confirmed by incorporating them into complete bottom-emitting VCSEL's with highly reflecting mirrors, resulting in threshold voltages as low as $1.49 \mathrm{~V}$ [11]. In addition, we found that the p-type top contact, especially in proton implanted regions, was a major contributor to the parasitic series resistance of top-emitting VCSEL's. This resistance was reduced by incorporating delta-doping layers in order to increase the contact doping and make it less susceptible to partial compensation from implant damage.

The epitaxial structure used for the high-efficiency topemitting InGaAs/AlGaAs VCSEL's reported in this letter is shown in Fig. 1. The structure was grown by MBE on an n-type GaAs substrate. The growth began with 33 periods of an n-type AlGaAs mirror with a high-reflectivity band nominally centered at $980 \mathrm{~nm}$, with a computed reflectivity of $99.8 \%$. The mirror layer composition was varied from $\mathrm{Al}_{0.1} \mathrm{Ga}_{0.9}$ As to $\mathrm{Al}_{0.9} \mathrm{Ga}_{0.1}$ As by cyclically changing the group III effusion cell temperatures in a complementary manner without any cell shuttering [10]. The first 25 periods of the mirror stack were doped with 


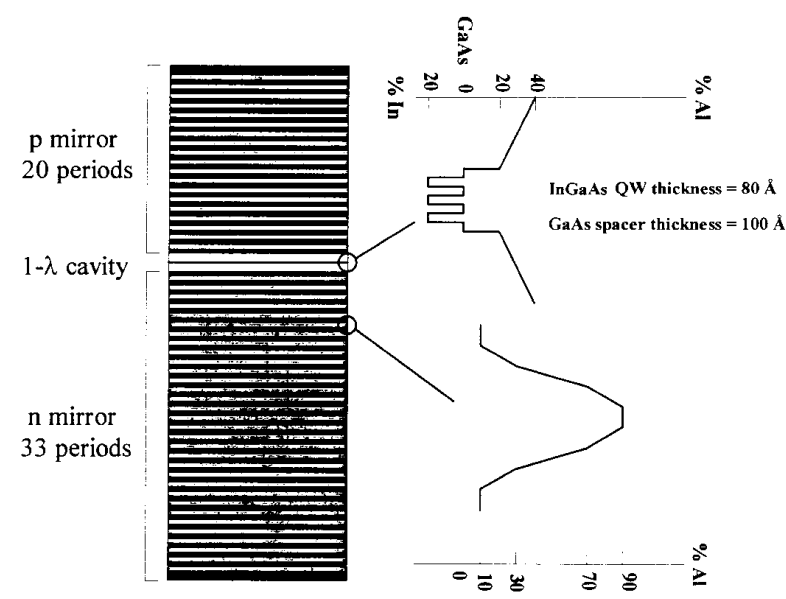

Fig. 1. Epitaxial structure of the VCSEL used for this study. The alloy composition variation for the graded confinement cavity and one period of the piecewise linearly graded mirrors are shown, with an uncalibrated position scale.

an activated $\mathrm{Si}$ concentration estimated at $2.0 \times 10^{18}$ $\mathrm{cm}^{-3}$. The remaining eight periods of the mirror stack were doped at a slightly lower concentration of $1.5 \times 10^{18}$ $\mathrm{cm}^{-3}$ to reduce free carrier absorption and scattering losses in the vicinity of the laser cavity, where the electric field is stronger. Following the n-type mirror a one-wavelength cavity was grown containing three 80 - $\AA$-thick $\mathrm{In}_{0.2} \mathrm{Ga}_{0.8}$ As strained quantum wells separated by 100 - $\AA$ GaAs barriers, as shown in Fig. 1. This quantum-well active region was clad on both sides by $\mathrm{Al}_{x} \mathrm{Ga}_{1-x} \mathrm{As}$ graded from $x=0.4$ at the mirrors to $x=0.2$ at the 100 - $\AA$ GaAs barrier outside the quantum wells. The n-type doping was gradually decreased in this graded AlGaAs cladding from $1.5 \times 10^{18} \mathrm{~cm}^{-3}$ at the mirror to $1 \times 10^{17}$ $\mathrm{cm}^{-3}$ near the quantum wells. The p-type Be-doping of the cladding above the quantum wells mirrored the n-type doping profile. After approximately $97 \%$ of the cavity was grown the growth was interrupted, and the reflectivity spectrum of the sample after cooling to room temperature was measured in an auxiliary ultra-high-vacuum analysis chamber. This measurement was used to make minor adjustments to the mirror and cavity thicknesses [12]. This provides accurate wavelength control at the center of the wafer, but wavelengths vary radially due to spatially nonuniform growth rates. Subsequently, the remainder of the cavity and a 20-period p-type top mirror with a computed reflectivity of $98.6 \%$ was grown. The estimated active Be-doping concentration in the eight periods closest to the cavity was $1.5 \times 10^{18} \mathrm{~cm}^{-3}$ and was increased to $3.0 \times 10^{18} \mathrm{~cm}^{-3}$ in the remaining 12 periods. Finally, a thin, heavily doped GaAs cap was grown that includes delta-doping spikes near the surface.

The material was fabricated into top-emitting VCSEL's by first defining, by liftoff, a rectangular p-type $\mathrm{Be}-\mathrm{Au}$ contact with a $10-\mu \mathrm{m}$-diameter circular aperture. This metallization was kept thin, at approximately $1500 \AA$, so that protons could later be implanted through it without excessive energy loss. A full-surface n-type $\mathrm{Ge}-\mathrm{Au}-\mathrm{Ni}-\mathrm{Au}$ contact was evaporated onto the backside of the wafer. Then 9- $\mu \mathrm{m}$-high $\times 12-\mu \mathrm{m}$-diameter photoresist dots centered about the $10-\mu \mathrm{m}$-diameter lasing aperture were patterned. These served as an implant mask for a deep proton implant designed to compensate the p-type mirror just above the active region. The $1-\mu \mathrm{m}$ overlap of the top contact metal into the unimplanted region was intended to improve the contact resistance. Individual lasers were isolated using a 5- $\mu$ m-deep wet etch around the contact to remove the conducting layers above the implant between devices. Lastly, the sample was rapid thermal annealed at $420^{\circ} \mathrm{C}$ for $30 \mathrm{~s}$ to alloy the contacts.

The devices were characterized p-side up on a probe station at room temperature. No special heatsinking or packaging was employed. $\mathrm{CW}$ light-current and voltage-current measurements were made using a commercial laser diode controller and a calibrated silicon photodiode. Initially, most of the devices that lased at 965-945 nm had 10\%-11\% maximum power conversion efficiencies. Selected devices were then burned in at a fixed current for up to 10 hours. The output power versus time curve for a representative device biased at $10 \mathrm{~mA}$ is shown in Fig. 2. At this fixed current, the output power increased by $1 \%$ in $15-30$ min with a diminishing but still positive rate of increase up to several hours. These burnins decreased the threshold current but slightly increased the voltage drop for a given current. The net result, however, was to increase the maximum power conversion efficiency, often to greater than $12 \%$. We believe that the burn-in anneals some of the implant damage, reducing the threshold current either by reducing nonradiative recombination traps near the active region or by reducing optical absorption and scatter [13].

The measured characteristics of a VCSEL lasing at 961 $\mathrm{nm}$ with a maximum power conversion efficiency of $12.7 \%$ are shown in Fig. 3. The device has a threshold current $I_{t h}$ of $4.0 \mathrm{~mA}\left(3.5 \mathrm{kA} / \mathrm{cm}^{2}\right)$ and a threshold voltage of $2.05 \mathrm{~V}$. A large range of high-efficiency operation, ideally in a single mode, is desirable for many applications. The power conversion efficiency of this device exceeds $10 \%$ over a 6.4-mA current range, from $4.8 \mathrm{~mA}\left(1.2 I_{t h}, 1.1 \mathrm{~mW}\right.$ output) to $11.2 \mathrm{~mA}\left(2.8 I_{t h}, 3.6 \mathrm{~mW}\right.$ output $)$. The laser operates in a single mode up to $2.1 \mathrm{~mW}$ output power before an additional higher-order transverse mode begins lasing at $7.3 \mathrm{~mA}$. The maximum power conversion efficiency occurs at $5.7 \mathrm{~mA}$ and an output power of $1.7 \mathrm{~mW}$, where the device is operating in a single mode with a transverse mode suppression of $\geq 40 \mathrm{~dB}$. We believe that $12.7 \%$ is the highest single-mode power conversion efficiency for VCSEL's of any type. The maximum power conversion efficiency of other high-efficiency VCSEL's has coincided with high-order mode lasing and exhibited size-dependent power conversion efficiencies, with the highest values for 20- $\mu \mathrm{m}$-diameter gain-guided [8] and index-guided [2] VCSEL's. We likewise anticipate higher power conversion efficiencies for larger, multimode devices. 


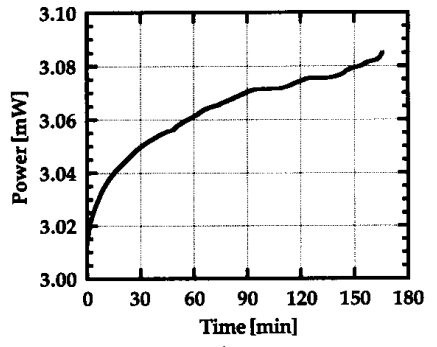

Fig. 2. A typical output power versus time curve during initial burn-in of a VCSEL at a fixed current of $10 \mathrm{~mA}$. Improvements in power conversion efficiencies were observed for all lengths of burn-in times up to a maximum recorded duration of $10 \mathrm{~h}$. The slight fluctuations in the curve may be due to changes in ambient temperature.

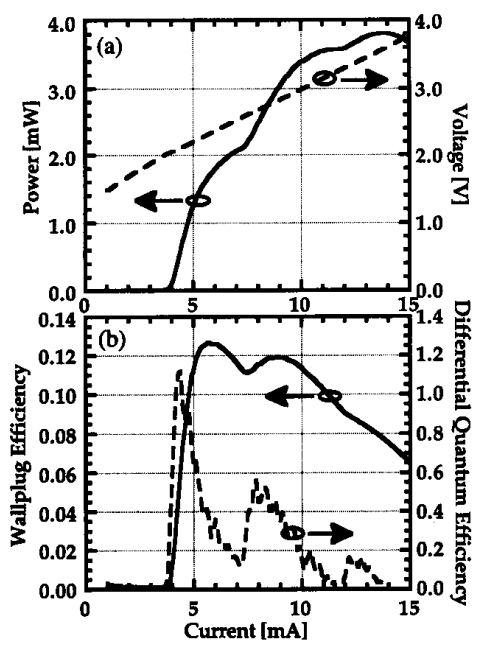

Fig. 3. $\mathrm{CW}$, room temperature characteristics of a vertical-cavity topsurface-emitting laser with $12.7 \%$ power conversion efficiency. Curves indicate (a) light (solid) and voltage (dashed), and (b) power conversion (solid) and differential quantum $\eta=(1 / h \nu)(d P / d I)$ (dashed) efficiencies.

A simple analysis of the contributions to power consumption in this device indicates that the high threshold current now rivals the series resistance as a major limitation to higher single-mode power conversion efficiency in these VCSEL's. The differential resistance of the voltage versus current curve shown in Fig. 3(a) is nearly constant above threshold with a value of $156 \Omega$. At the maximum power conversion efficiency of $12.7 \%$ at $5.7 \mathrm{~mA}$ where the output power is $1.7 \mathrm{~mW}$ for a total input power of 13.1 $\mathrm{mW}$, the power dissipated by the series resistance is 5.1 $\mathrm{mW}$, leaving the remaining $6.3 \mathrm{~mW}$ to be attributed to optical losses, spontaneous emission, and nonradiative recombination. Since the differential quantum efficiency is quite high from threshold to $5.7 \mathrm{~mA}$, the largest portion of the input power, $5.6 \mathrm{~mW}$, is consumed in reaching threshold. Thus the threshold must also be reduced for increased efficiency. Two factors contribute to the high threshold of the present device: The quantum wells have been designed for lasing at $980 \mathrm{~nm}$, while the portion of the wafer processed for this study had a maximum cavity- mode wavelength of $965 \mathrm{~nm}$. Also, the output coupler reflectivity has been chosen to be relatively low. Higher top-mirror reflectivity would decrease the threshold at the expense of optical efficiency. The series resistance can also be further reduced. We estimate an upper limit of 30 $\Omega$ for vertical transport through the p-type mirror from previous measurements of similar variably doped p-type mirror stacks [10]. Thus the majority of the resistance comes from the contacts and n-type mirror and lateral current flow in the implanted region, indicating further improvements can be made. Based on this simple analysis, we believe that power conversion efficiencies in excess of $20 \%$ can be attained in top-emitting VCSEL's by reducing the threshold current densities by $50 \%\left(1.7 \mathrm{kA} / \mathrm{cm}^{2}\right)$.

In conclusion, we have demonstrated top-emitting In$\mathrm{GaAs} / \mathrm{AlGaAs}$ VCSELs with $\mathrm{CW}$ power conversion efficiencies as high as $12.7 \%$ at $1.7 \mathrm{~mW}$ output power in a single mode. This increased efficiency has been accomplished by incorporating low resistance, piecewise linearly graded mirrors and delta-doped contact layers into a structure that was fabricated into gain-guided devices using deep proton implants. The series resistance of these VCSEL's has been reduced to such a level that the power consumed in reaching threshold equals the resistive power dissipation for single-mode operation. Continued improvements in device efficiency can be expected with reduced absorption and scattering losses and reduced nonradiative recombination, as well as further improvements in device resistance.

\section{ACKNOWLEDGMENT}

The authors would like to thank S. Kilcoyne, K. Killeen, and J. Nevers for technical assistance, and K. Choquette, J. Hohimer, A. Owyoung, and J. Scott for useful discussions and comments.

\section{REFERENCES}

[1] D. Welch, R. Craig, W. Streifer, and D. Scifres, "High reliability, high power, single mode laser diodes," Electron. Lett., vol. 26, pp. 1481-1483, 1990.

[2] M. G. Peters et al., "High wallplug efficiency vertical-cavity surface-emitting lasers using lower barrier DBR mirrors," Electron. Lett., vol. 29, pp. 170-172, 1993.

[3] M. G. Peters et al., "High-efficiency vertical-cavity surface-emitting lasers with low barrier p-type mirrors," in Proc. Conf. Lasers and Electro-Optics, OSA, Baltimore, MD, 1993, vol. 11, p. 136.

[4] Y. H. Lee, B. Tell, K. Brown-Goebeler, J. L. Jewell, and J. V. Hove, "Top-surface emitting GaAs four-quantum-well lasers emitting at $0.85 \mu \mathrm{m}, "$ Electron. Lett., vol. 26, pp. 710-711, 1990.

[5] W. Nakwaski, M. Osinski, and J. Cheng, "Spreading resistance in proton-implanted vertical-cavity surface-emitting diode lasers," Appl. Phys. Lett., vol. 61, pp. 3101-3103, 1992.

[6] J. A. Lott and R. P. Schneider, "Electrically injected visible (639-661 nm) vertical cavity surface emitting lasers," Electron. Lett., vol. 29, pp. 830-831, 1993.

[7] M. E. Warren et al., "On-axis far-field emission from two-dimensional phase-locked vertical cavity surface-emitting laser arrays with an integrated phase-corrector," Appl. Phys. Lett., vol. 61, pp. 1484-1486, 1992.

[8] K. Kojima, et al., "High power GaAs/AlGaAs vertical cavity top-surface-emitting lasers," in Proc. 13th IEEE Int. Semiconductor Laser Conf., Tokyo, Japan, 1992, paper PD-2.

[9] D. P. Bour and A. Rosen, "Optimum cavity length for high conversion efficiency quantum well diode lasers," J. Appl. Phys., vol. 66 , pp. $2813-2818,1989$. 
[10] S. A. Chalmers, K. L. Lear, and K. P. Killeen, "Low resistance wavelength-reproducible p-type (Al,Ga)As distributed Bragg refiectors grown by molecular beam epitaxy," Appl. Phys. Lett., vol. 62 , pp. $1585-1587,1993$.

[11] K. L. Lear, S. A. Chalmers, and K. P. Killeen, "Low threshold voltage vertical cavity surface-emitting laser," Electron. Lett., vol. 29 , pp. $584-586,1993$.
[12] S. A. Chalmers and K. P. Killeen, "Method for accurate growth of vertical-cavity surface-emitting lasers," Appl. Phys. Lett., vol. 62 , pp. 1182-1184, 1993.

[13] T. Numai et al., "High electronic-optical conversion efficiency in a vertical-to-surface transmission electro-photonic device with a vertical cavity," IEEE Photon. Technol. Lett., vol. 5, pp. 136-138, 1993.

\title{
Lateral and Longitudinal Mode Discrimination in Index-Guided Circular Ring Semiconductor Lasers
}

\author{
R. F. Nabiev, D. Francis, and C. J. Chang-Hasnain
}

\begin{abstract}
We present the first analysis of the mode structure and thresholds of index-guided ring lasers, and on longitudinal mode discrimination of $\boldsymbol{X}$-junction-coupled ring lasers. Submilliampere thresholds can be achieved with single-lateral-mode ring lasers having small refractive index steps. In addition, strong longitudinal mode discrimination can be obtained with ring lasers of different cavity lengths coupled via an $X$ junction.
\end{abstract}

EMICONDUCTOR ring lasers are promising be$D$ cause they offer a number of important advantages over other laser structures. First, the cavity lengths are precisely defined, which is desirable for short-pulse and microwave signal generation. The topology allows for efficient use of real estate. In addition, using a surface-emitting output coupler, the ring lasers provide an elegant alternative to low-threshold surface-emitting lasers. A number of papers have been published on ring lasers [1]-[3]. Most ring lasers have been made with deeply etched structures to provide stronger waveguiding. However, such structures exhibit high thresholds due to surface recombination, etching damages, and scattering losses. They also lase in multiple lateral modes because of the large index step $(\Delta n \sim 2.5)$. Ring lasers with small index steps $(\Delta n=0.1-0.2)$ using, for example, ridge waveguides or buried heterostructures are free from these shortcomings and thus should exhibit better lateral-mode confinment and lower threshold currents. In this paper we present an analysis of mode structure and thresholds of ring semiconductor lasers with small index steps. We show that, contrary to what is commonly believed, low threshold

Manuscript received May 28, 1993; revised June 15, 1993. This work was supported by the NSF Young Investigator Program under Grant ECS-92 57209, and by a Powell Research Award. The work of D. Francis was supported by AT \& T.

The authors are with the E. L. Ginzton Lab and the Department of Electrical Engineering, Stanford University, Stanford, CA 94305-4085.

IEEE Log Number 9211562. for the fundamental radial mode and strong suppression of higher order lateral modes can be simultaneously attained with such lasers. In addition, we explore the possibility of longitudinal-mode discrimination using different types of $X$-junction-coupled ring lasers.

Ridge-waveguide ring lasers can be described in terms of effective index approximation, with a certain profile of refractive index $n(r)$ that depends on the radius. An electromagnetic field of form $A(r) r^{-1 / 2} \exp (-i M \varphi+$ $i \omega t)$ obeys the wave equation $(M \gg 1)$

$$
\frac{\partial^{2} A}{\partial r^{2}}-k_{0}^{2}\left(\frac{R^{2} n_{\mathrm{eff}}^{2}}{r^{2}}-n^{2}(r)\right) A=0
$$

where $k_{0}=\omega / c$ is the wave vector of photons in a vacuum, $R$ is the outer diameter of the ring, $n_{\text {eff }}$ is the analog of modal refractive index in planar waveguides, and $M=2 \pi R n_{\text {eff }} / \lambda_{0}\left(\lambda_{0}\right.$ is the wavelength in a vacuum). The $M$ value ( $\rightarrow 1$ ) characterizes the number of wavelengths along the circumference of the ring. Fig. 1(a) shows intensity distributions of the three lowest order modes of a $2-\mu \mathrm{m}$-wide ring waveguide with $100-\mu \mathrm{m}$ radius and an index step $\Delta n=0.1$. The fundamental mode ( $N$ $=1$ ) is shifted to the outer boundary of the ring, whereas the higher order modes are shifted to the inner radius of the ring. Fig. 1(b) shows the radial intensity profiles of the fundamental modes of two waveguides with different radii: $R=50 \mu \mathrm{m}$ and $R=150 \mu \mathrm{m}$. The mode profile is less shifted to the outer boundary for the ring with a larger radius because the $1 / r^{2}$ term in (1) is flatter inside the waveguide region. Further increasing of the width of the ring does not significantly change the profile and the effective refractive index $n_{\text {eff }}$ of the fundamental mode.

The bending of the waveguide causes the electromagnetic field to tunnel through a potential barrier, which in turn leads to inherent mode leakage [see the solid line for $R=50 \mu \mathrm{m}$, Fig. 1(b)]. We estimate the bending losses for 